\title{
La Investigación Arqueológica en el Palacio San José - Museo y Monumento Histórico Nacional “Justo José de Urquiza” (Entre Ríos, Argentina)
}

Sandra Guillermo

Ministerio de Cultura de la Nación y Arqueología \& Consultoría (gestión, investigación y patrimonio). Alsina 465, $5^{\circ}$ piso (CP C1087AAE), Ciudad Autónoma de Buenos Aires, Argentina. E-mail: sandra.guillermo@gmail.com

Recibido: 31 de marzo de 2020

Aceptado: 23 de junio de 2020

\begin{abstract}
Resumen
Durante el siglo XIX el Palacio San José constituyó la residencia familiar de Justo José de Urquiza, un prestigioso político, militar y hacendado argentino, gobernador de la provincia de Entre Ríos y Presidente de la Confederación Argentina entre 1854 y 1860. La investigación arqueológica realizada en el predio que rodea dicha residencia fue la primera experiencia efectuada en el lugar desde esta disciplina. Articuló la investigación científica académica con una fuerte participación de la comunidad, a través de asociaciones colaborativas con instituciones universitarias asentadas en Concepción del Uruguay y otras instituciones locales, lo cual le otorgó a la práctica arqueológica desarrollada una característica particular. El objetivo general fue identificar y recuperar evidencia presente en tres áreas que rodean al Palacio San José durante el momento de ocupación del sitio en el siglo XIX. Mediante la excavación arqueológica se hallaron restos estructurales, tales como una vereda de ladrillos artesanales, los cimientos de un mirador con un patio con canteros, así como también restos artefactuales (botellas, vidrios, pipas, clavos, etc.) de la vida cotidiana del siglo XIX provenientes de la excavación de un basural. En este artículo se presentan los principales lineamientos de la investigación y los resultados generales alcanzados luego de realizados los trabajos de campo y de gabinete.
\end{abstract}

PALABRAS CLAVE: Lago artificial; Basural; Siglo XIX.

\section{The Archaeological Research at San José Palace - Museum and National Historical Monument "Justo José de Urquiza" (Entre Ríos, Argentina)}

\begin{abstract}
During the 19th century, the San José Palace was Justo José de Urquiza's family residence. He was a prestigious Argentine politician, military man, and landowner, and he also served as Governor of Entre Ríos province and President of the Argentine Confederation between 1854 and 1860 . The archaeological research in the property surrounding the residence was the first experience carried out in this place. Articulated
\end{abstract}


scientific research with strong community participation through partnerships with Universities located at Concepción del Uruguay, and other local institutions, gave this archaeological practice a particular characteristic. The general goal was to identify and recover evidence from three areas surrounding San José Palace, which belonged to the site's occupation in the 19th century. Archaeological excavations yielded several structural remains, such as an artisanal brick sidewalk, and the foundations of a viewpoint that included a courtyard with stone flowerbeds. Also, material remains of everyday life of the 19th century (bottles, glass, pipes, nails, etc.) were found during a dump excavation. This article presents the main research guidelines and the general results achieved after field and laboratory work.

KEYWORDS: Artificial lake; Dump; $19^{\text {th }}$ Century.

\section{Introducción}

El Palacio San José constituyó, durante la segunda mitad del siglo XX, la residencia del general Justo José de Urquiza, quien en el año 1848 inició, en un predio de alrededor de 2.500 hectáreas, la construcción del casco de su estancia, ubicada a $30 \mathrm{~km}$ de la ciudad de Concepción del Uruguay, en la Provincia de Entre Ríos (Figura 1).

Entre los años 1861 y 1868 Urquiza construyó un poco más allá del jardín posterior de su residencia, un lago artificial de grandes dimensiones $(180 \mathrm{~m}$ de largo por $120 \mathrm{~m}$ de ancho) con fines recreativos y a continuación de éste, en la misma línea, un corral de dimensiones similares en donde se llevaban a cabo actividades relacionadas con la hacienda. Ambos estaban insertos en un predio abierto y extenso que fue a su vez intensamente ocupado también por otras construcciones y actividades vinculadas a diferentes fines - plantaciones de árboles frutales, caballerías, herrería, almacén de ramos generales- (Benvenuto, 1988; Cerrudo, 2000).

La ausencia de estudios arqueológicos en el predio que rodea a la residencia y su conjunto edilicio, ha motivado llevar a cabo la presente investigación. La misma tuvo como objetivo principal poder identificar, recuperar y salvaguardar el patrimonio arqueológico aún presente en el lugar, con la finalidad de reconstruir y conocer diferentes aspectos vinculados con su secuencia de ocupación y con la vida cotidiana de sus ocupantes durante el siglo XIX.

En esta nota se presentan los resultados alcanzados por dicha investigación, realizada entre los años 2014 y 2017, la cual fue coordinada por la autora como arqueóloga del Ministerio de Cultura de la Nación. La residencia y todo su espacio circundante, declarados Monumento Histórico Nacional en el año 1935, hoy en día constituyen un Museo Nacional que integra la Dirección Nacional de Museos del Ministerio de Cultura de la Nación. La investigación articuló el trabajo científico-académico con una fuerte participación de la comunidad a través de asociaciones colaborativas que permitieron su realización, así como también una intensa participación de alumnos universitarios en las diferentes actividades efectuadas.

Al momento de iniciarse la investigación proyectada se suscitó el inconveniente de no poder disponer de los recursos económicos y logísticos necesarios. Esto llevó a una detención inicial de las acciones, permaneciendo al borde de la cancelación los trabajos de campo atinentes al proyecto, ya que no podían ser solventados desde el Ministerio de Cultura de la Nación y tampoco desde el Museo. Sin embargo, al evaluarse diferentes alternativas posibles, el Director del Museo y la coordinadora del área del parque propusieron llevar a cabo asociaciones colaborativas (a través de aportes 


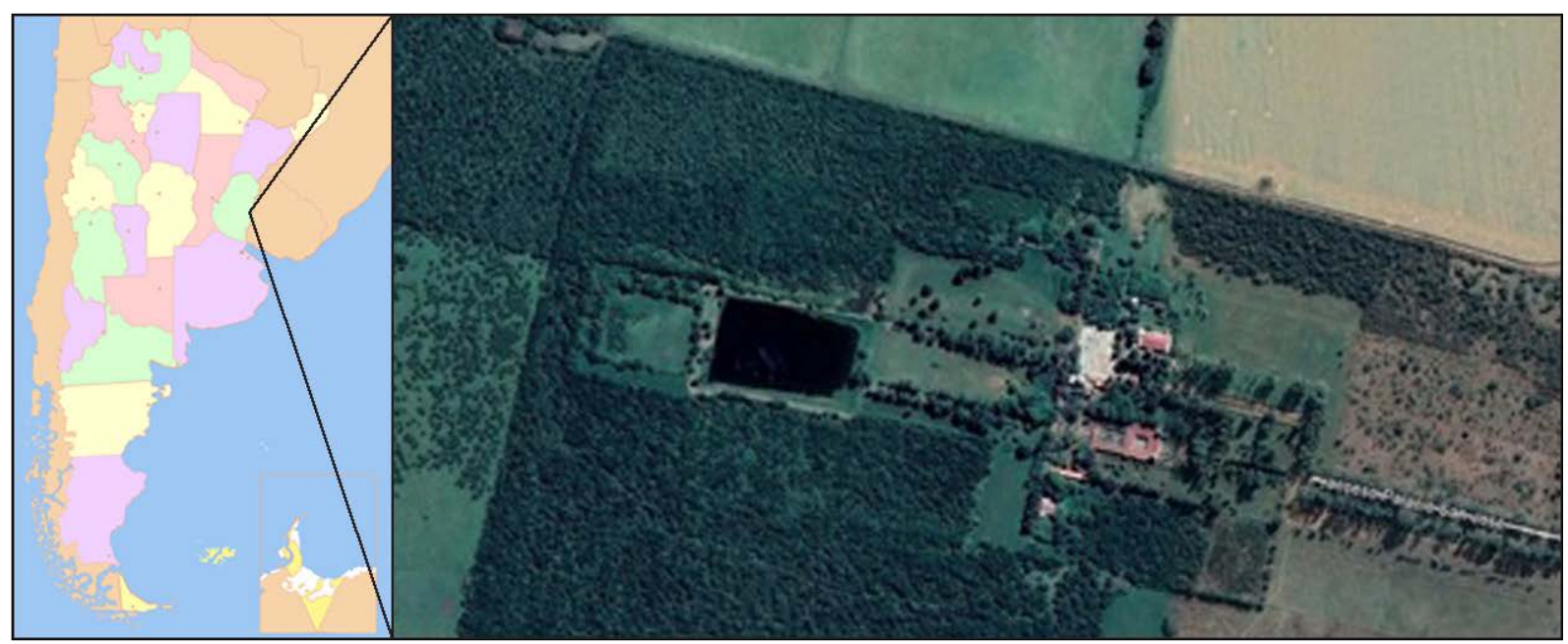

Figura 1. Localización y vista aérea del predio en el que se encuentra el Palacio San José - Museo y Monumento Histórico "Justo José de Urquiza" en la provincia de Entre Ríos - Argentina (Google Earth - Image 202 CNES/AIRBUS).

económicos, técnicos, profesionales, voluntariados universitarios y herramientas comunicacionales) con diferentes instituciones locales, lo cual permitió finalmente la realización del proyecto.

Una vez iniciada la investigación y previo al comienzo del trabajo arqueológico, se realizó un relevamiento de la información general localizada en el Archivo y Biblioteca del Museo, vinculada a la historia del lugar y de sus habitantes, con la finalidad de contextualizar adecuadamente dicho trabajo. Luego, se realizó una prospección general de todo el predio para reconocer, identificar y establecer el potencial arqueológico de los diferentes espacios. Los resultados obtenidos, conjuntamente con la sistematización de los datos del relevamiento previo, permitieron establecer tres espacios a intervenir: el área que conforma el perímetro del lago artificial, el sector oeste de dicho perímetro y un sector localizado en el área que rodea la residencia, el cual habría sido utilizado para el descarte de basura, durante los años en que la familia Urquiza ocupó el lugar.

El registro arqueológico identificado y recuperado está conformado tanto por evidencia de tipo estructural (sectores y fragmentos del muro perimetral del lago, cimientos de un mirador del siglo XIX, un patio y una vereda de ladrillos de confección artesanal), como de tipo artefactual, vinculado con la vida cotidiana (botellas de gres, lozas, metales, restos óseos de animales consumidos, pipas, etc.).

A continuación se describirán los principales aspectos del Palacio San José, luego se hará mención al proyecto de investigación y a las asociaciones colaborativas establecidas. Posteriormente, el texto se centrará en el trabajo arqueológico realizado en tres de las áreas establecidas a partir de la prospección inicial y en la identificación y recuperación de la evidencia hallada en cada una de ellas, así como también en la metodología empleada. Finalmente, se mencionarán los resultados obtenidos y las consideraciones finales a las cuales se arribó a partir de todo lo enunciado a lo largo del texto.

\section{El Palacio San José}

Tal como se ha mencionado anteriormente, el Palacio San José, hoy día Museo y Monumento Histórico "Justo José de Urquiza", constituyó durante la segunda mitad del siglo XIX la residencia del general Justo José de Urquiza, un prestigioso político 
y militar, comerciante y hacendado argentino, gobernador de la provincia de Entre Ríos y presidente electo de la Confederación Argentina, entre los años 1854 y 1860 (Benvenuto, 1988).

En el año 1848 inició la construcción del casco de su estancia, en ese entonces de más de 2.500 hectáreas, ubicada a $30 \mathrm{~km}$ de la ciudad de Concepción del Uruguay, en la provincia de Entre Ríos. Arquitectos italianos diseñaron su amplia residencia, con alrededor de 38 habitaciones, dos patios localizados en un eje de Este a Oeste e instalaciones específicas destinadas a diferentes actividades tales como: herrería, palomar, pajareras, caballerizas y almacén de ramos generales, así como también una capilla. Todo ello rodeado por jardines y un extenso parque con plantaciones de árboles frutales, aljibes y hasta un pequeño zoológico (Cerrudo, 2000).

Entre los años 1861 y 1868, Urquiza mandó a construir un lago artificial, ubicado luego del jardín posterior, que impacta por su gran dimensión (120 m de ancho por 180 $\mathrm{m}$ de largo), por su muro perimetral elaborado sobre un terraplén artificial de arcilla recubierto de mampostería y por el empleo de un sofisticado sistema de cañerías y bombas (compradas en Estados Unidos), cuyo propósito era mantener de manera permanente cierto nivel de agua que ingresaba desde una laguna cercana que se encontraba vinculada con el río Gualeguaychú. Este lago fue construido para el ocio y el recreo, contando además con un barco tipo Pailebot a vapor, traído especialmente de Inglaterra y con embarcaciones menores para su disfrute (Cerrudo, 2000).

Unos metros más hacia el Oeste, en línea con el lago y hacía el final del mismo, se localizaba un corral (Figura 2), conformado también por un espacio de importantes dimensiones ( $130 \mathrm{~m}$ de largo por $90 \mathrm{~m}$ de ancho) y rodeado, al igual que el lago, por un terraplén artificial de arcilla recubierto con mampostería. Allí se apartaba la hacienda y se realizaban diversas actividades relacionadas con el manejo de los animales (Cerrudo, 2000).

En abril del año 1870, después que el general Justo José de Urquiza es asesinado en el interior de su vivienda, la residencia es abandonada por su familia, sin volver a ser formalmente ocupada. En el año 1935 es creado allí el Museo Nacional, siendo el Palacio San José declarado entonces Monumento Histórico Nacional, mediante la Ley $\mathrm{N}^{\circ} 12.261$ de ese mismo año (https://museourquiza.cultura.gob.ar/info/el-palacio/; Boletín oficial $n^{\circ} 12394$ ).

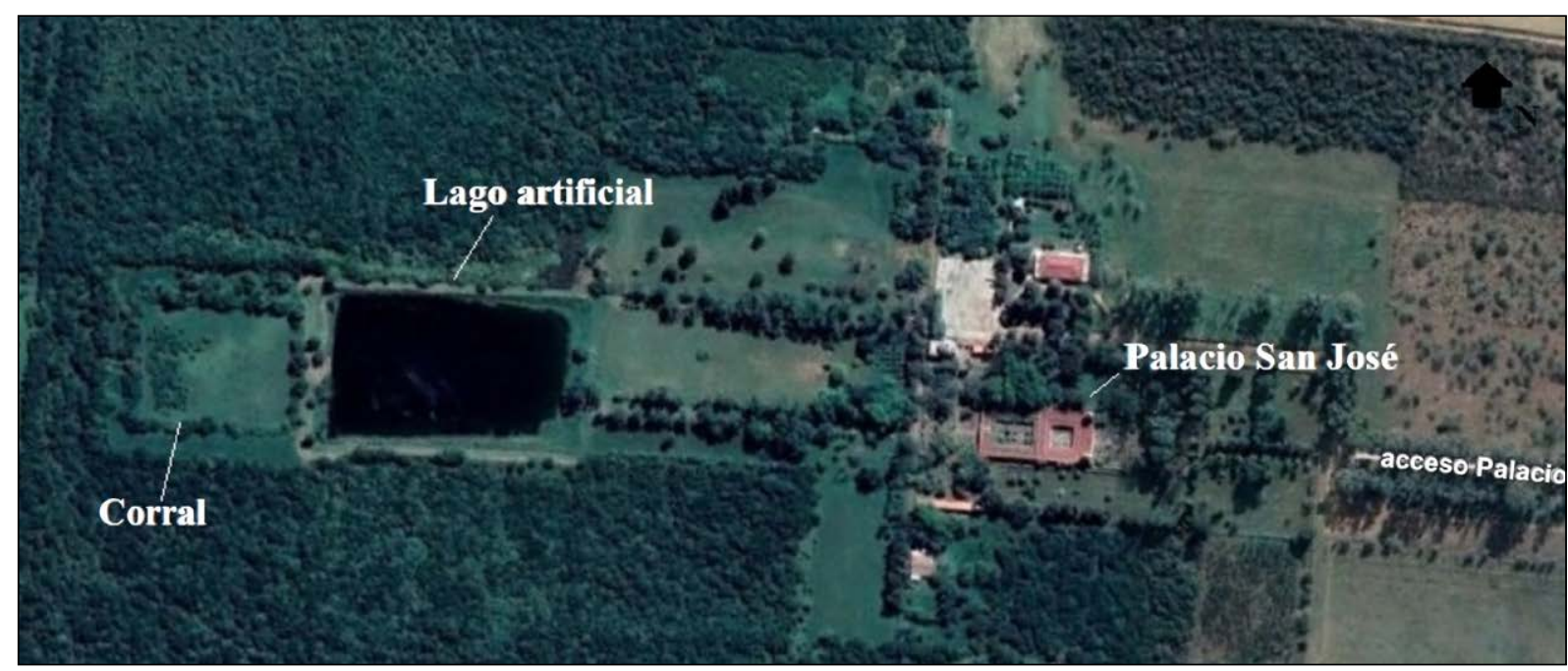

Figura 2. Predio actual del Museo. Sobre un mismo eje, el palacio San José, el lago artificial y el corral (Google Earth - Image 202 CNES/AIRBUS). 


\section{El proyecto de investigación y las asociaciones colaborativas}

La investigación arqueológica, coordinada por la autora, formó parte de un proyecto mayor de puesta en valor del parque del lago, desarrollado y llevado adelante por el propio museo, con el propósito de recuperar la historia del espacio que rodea la residencia y el lago artificial, promoviendo así la revalorización del lugar, e intentando integrar, interpretar y exhibir sus componentes en el marco de su secuencia de ocupación.

Tal como se ha mencionado, el inconveniente de no poder disponer de recursos económicos y logísticos destinados a la investigación llevó casi a la anulación del proyecto. Sin embargo, al analizarse y evaluarse diferentes alternativas posibles, el director del museo y la coordinadora del área del parque decidieron efectuar primeramente un mapeo general de la comunidad de Concepción del Uruguay y de los vínculos establecidos con el museo, para poder identificar entre todos ellos posibles "actores" sociales estratégicos (Tapella, 2007), que quisieran y pudieran participar de este proyecto y dar forma así a una red de colaboración en diferentes niveles. Esto permitió conformar una serie de asociaciones colaborativas, las cuales posibilitaron llevar a cabo la investigación arqueológica en el lugar y la ejecución de las tareas. Estas asociaciones colaborativas involucraron y relacionaron a la comunidad con el proyecto de diferentes maneras: mediante recursos económicos, técnicos, profesionales, voluntariados universitarios y herramientas comunicacionales.

La ciudad de Concepción del Uruguay congrega una gran cantidad de centros universitarios y de formación terciaria. A aquellos que poseían carreras vinculadas o interesadas en el patrimonio se les ofreció la posibilidad de que cierta cantidad de alumnos pudiera participar de esta investigación, como práctica universitaria. De esta manera, la Universidad de Concepción del Uruguay, la Universidad Tecnológica Nacional y la Universidad Autónoma de Entre Ríos, a través de sus carreras de Museología, Arquitectura, Ingeniería en Materiales e Historia, formaron parte de la investigación mediante la participación de sus alumnos como voluntarios del equipo técnico que realizaría las excavaciones en el Palacio San José. Se les solicitó como requisito que fuesen alumnos regulares y que viviesen en la ciudad de Concepción del Uruguay o sus alrededores, para que de esta manera los participantes pudiesen vivir una experiencia de trabajo con su propio patrimonio local.

Se tenía muy poco conocimiento acerca de si la propuesta iba a despertar interés en estudiantes universitarios. Sin embargo, se pudo observar con sorpresa y con mucha satisfacción que así fue. En la primera convocatoria se presentaron aproximadamente diez alumnos por cada una de las carreras universitarias, de los cuales se seleccionaron cuatro de Museología y tres de cada una de las restantes disciplinas. Al año siguiente el número de inscriptos en las listas se había incrementado un poco más y en el último año de trabajo, la cantidad aumentó considerablemente, existiendo además una lista complementaria de alumnos en espera.

El proyecto tuvo repercusión no solo en el ámbito académico, sino además en la comunidad en general, promoviendo el interés en la investigación arqueológica. Una vez iniciado el trabajo, los visitantes que recorrían el palacio, así como los familiares y amigos de los estudiantes que colaboraron en las excavaciones, tuvieron también la posibilidad de acercarse a observar las actividades que se estaban llevando a cabo.

La colaboración de la Asociación de Amigos del Museo fue importante, tanto para distintos aspectos de la investigación como para la concreción del trabajo con los voluntarios universitarios, ya que aportó recursos económicos para proveerles de un viático diario y el traslado desde y hacia Concepción del Uruguay todos los días mientras se realizaba el trabajo de campo. Además proveyó los materiales necesarios para que 
cada uno de los integrantes tuviera sus propios elementos de trabajo. En cuanto a la conformación del equipo, también se convocó a participar a personas de la Municipalidad de Herrera y de los museos de los alrededores del Palacio (como el Museo de la Colonia de San Justo) y a profesionales y personal del propio Palacio San José.

Las asociaciones colaborativas se aplicaron también a otros aspectos de la investigación. Entre ellos estuvo, por ejemplo, la elaboración de un relevamiento planimétrico de todo el perímetro del lago artificial, que no se poseía, realizado por profesionales agrónomos de la Municipalidad de Herrera y de la Universidad Tecnológica Nacional. De esta manera, se pudo disponer de un plano donde poder localizar los diferentes puntos intervenidos en las excavaciones; además, el museo pudo contar con dicho relevamiento para su propio uso.

Con esta metodología de colaboración también se pudieron efectuar, una vez iniciados los trabajos, estudios detallados que mejoraron los resultados, dando más especificidad y profundidad a la información obtenida. La Universidad del Museo Social Argentino brindó de forma gratuita el análisis de las maderas halladas en excavación, realizado por profesionales de la carrera de Conservación y Restauración de Bienes Culturales. Asimismo, la Universidad Tecnológica Nacional (sede Concepción del Uruguay) ofreció su laboratorio de materiales, para que pudiesen llevarse a analizar allí las muestras de arcilla extraídas del perímetro del lago artificial, sobre el cual se asentó el muro que lo conforma y cuyos resultados fueron imprescindibles para entender la dinámica posterior de su desmoronamiento hacia el interior del lago.

Por otro lado, el Director del Palacio San José, además de otorgar el permiso a su personal para que algunos de ellos pudiesen colaborar en el trabajo de campo, brindó a los voluntarios material acerca de la historia del Palacio San José conjuntamente con una visita guiada. Acondicionó los espacios a intervenir y preparó un área donde llevar a cabo la capacitación inicial y las tareas básicas. Facilitó también la obtención de información $y$ datos de la historia oral a través de los relatos informales de empleados del museo con mucho conocimiento de la historia del sitio y contactó a personas del lugar que pudiesen aportar narraciones de otros tiempos, transmitidos por sus padres, abuelos, familiares, etc.

De esta manera, la participación ciudadana y del personal y profesionales del Palacio San José constituyó una parte fundamental de la investigación y recuperación del patrimonio arqueológico del lugar, dada la internalización y naturalización que se produjo en relación con esta actividad. Implicó insertar a la arqueología dentro de la esfera de lo público, considerándola en su sentido más amplio, a partir de la vinculación del trabajo arqueológico con la sociedad (Lleras, 2018) y teniendo presente que la arqueología concebida de este modo constituye no sólo una actividad académica, sino también una actividad social (Salerno, 2013) que va más allá de la divulgación del trabajo arqueológico y de los resultados obtenidos (Almansa Sanchéz, 2014).

\section{El trabajo arqueológico: áreas excavadas, metodología y resultados obtenidos}

La investigación arqueológica se inició en el año 2014 mediante la ejecución de una prospección inicial en el predio del parque del museo. Esta incluyó el recorrido de un área aproximada de $1 \mathrm{~km}^{2}$ observando y registrando las características del espacio natural y de sus principales rasgos, sin recolección superficial de restos materiales ni planteo de transectas. Se realizó también una revisión general de textos históricos y de fuentes documentales disponibles en el archivo y biblioteca de la institución. De esta manera, los datos obtenidos constituyeron la base en torno a la cual se plantearon las posteriores excavaciones arqueológicas. 
Mediante la prospección efectuada se identificaron y establecieron cuatro áreas con un alto potencial arqueológico: 1) el área conformada por un espacio de entre 2 y $5 \mathrm{~m}$ que comprende el perímetro del lago artificial, 2) el sector oeste del perímetro, 3) un área con una alta densidad de restos arqueológicos superficiales y en estratigrafía, ubicada hacía el lado sur del predio y 4) el área de un tajamar del siglo XIX. De todas ellas, esta última no fue considerada en esta parte de la investigación arqueológica, dado que implicaba la realización de otro tipo de acciones, además de las arqueológicas (Figura 3).

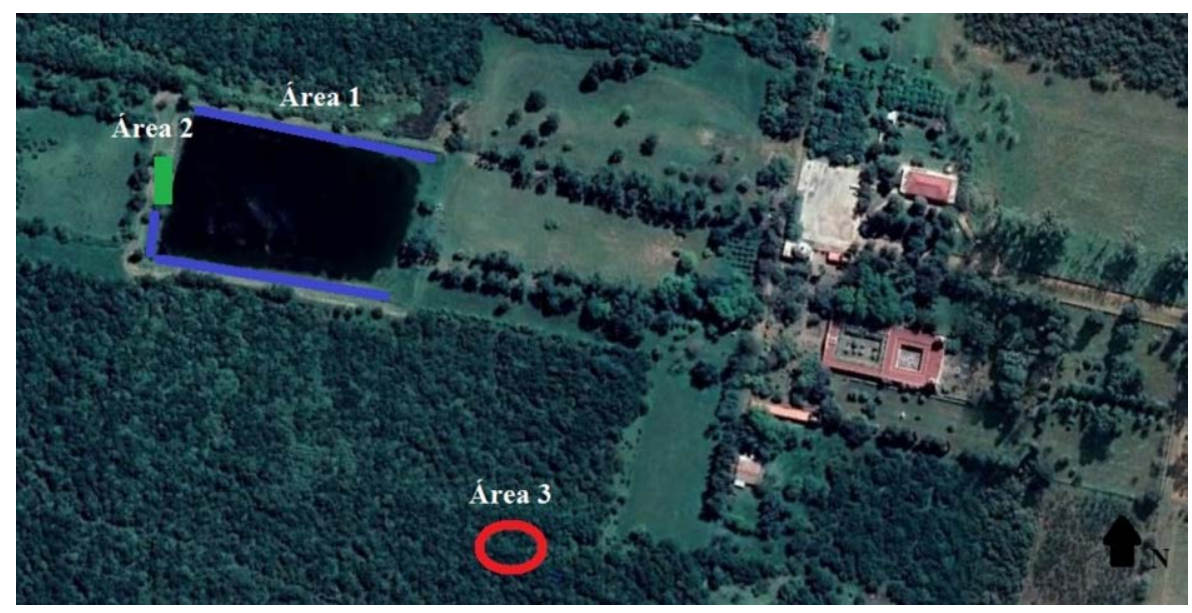

Figura 3. Ubicación de las tres áreas que fueron estudiadas arqueológicamente (Google Earth - Image 202 CNES/AIRBUS).

En función de estos resultados y de los datos obtenidos en el relevamiento histórico y documental, se planificaron las excavaciones arqueológicas, las que se realizaron en los años 2014, 2015 y 2016. En las tres oportunidades se constituyeron equipos de trabajo diferentes, mediante la modalidad de colaboración por voluntariado acordada con las distintas instituciones, según fuera mencionado anteriormente (Figura 4).

Los equipos conformados (entre 12 y 18 personas cada uno) recibieron antes de iniciar las actividades, una capacitación básica sobre los principales conceptos arqueológicos, la metodología de trabajo que se iba a implementar y el cronograma de las distintas actividades a realizar. Se les brindó además una visita guiada, efectuada por personal del museo y se les otorgó material impreso sobre la historia del lugar, para que conocieran el espacio en el que iban a trabajar con cierta profundidad y valorizaran la importancia que reviste a nivel histórico.

El objetivo general de la investigación arqueológica fue identificar y recuperar toda evidencia aún presente en el lugar, perteneciente al momento de ocupación del lugar en el siglo XIX. En cuanto a los objetivos específicos, éstos apuntaron a determinar y establecer las características morfológicas y constructivas del lago artificial y de su perímetro y determinar la forma y dimensión del mirador y de sus componentes asociados, localizados en el lado oeste de dicho perímetro. Asimismo, para el área que presentó una alta densidad de restos arqueológicos y que fuese definida a partir del resultado de los sondeos iniciales como un área de descarte, cronológicamente perteneciente al siglo XIX, el objetivo específico estuvo centrado en determinar su extensión, profundidad y composición.

De esta manera, la investigación arqueológica se llevó a cabo en las siguientes tres áreas y subáreas: 


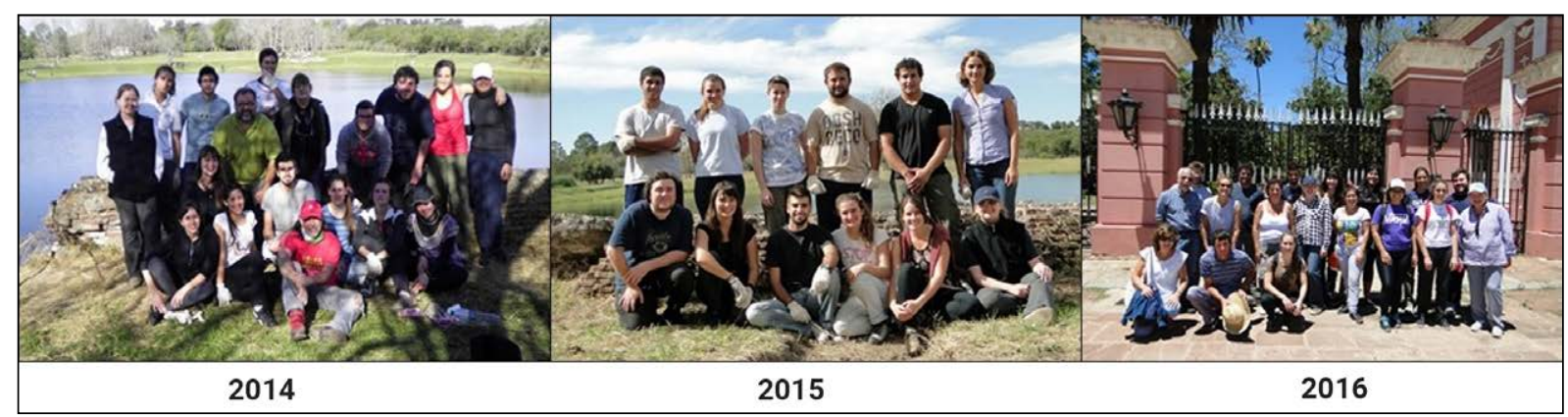

Figura 4. Equipos conformados a lo largo de los años de trabajo en el sitio.

\section{El perímetro del lago artificial}

En todo el espacio que rodea al lago artificial se efectuaron en diferentes puntos excavaciones de sondeos, cuadrículas y trincheras.

Cuando el lago fue construido, a mediados del siglo XIX, poseía en todo su perímetro un muro de ladrillos de confección artesanal ${ }^{1}$ (Castro, 1944), asentado sobre uno de los lados de un terraplén artificial compuesto por un sedimento arcilloso propio del lugar. Con el paso del tiempo, éste fue desmoronándose paulatinamente y cayendo hacia el interior del lago. En cuanto al terraplén y la mampostería que lo recubría aún están presentes, encontrándose al momento de la investigación por debajo de una capa de césped.

Una vez retirado el césped, el trabajo arqueológico sobre el terraplén permitió identificar en su parte superior la presencia de una vereda conformada por ladrillos de confección artesanal, conjuntamente con el cimiento de un muro divisor, que la transformaba en algo similar a una senda con boulevard (Tabla 1). De acuerdo a la información proporcionada por el área de Archivo del Palacio y por uno de los textos consultados (Castro, 1944), ésta era utilizada tanto por peatones, como por caballos y carruajes. Su estado de conservación varía de acuerdo a los sectores excavados. Los lados oeste y sur son los que presentan una mayor integridad y lo más frecuentemente observado en ellos es la fragmentación de la capa más superficial de los ladrillos, es decir la que se localiza inmediatamente por debajo del césped.

\section{1.a. El interior del lago}

La parte interna del lago también fue objeto de investigación, con el propósito de determinar la forma en que fue construido y los materiales que se utilizaron para ello. La información obtenida complementó los datos provenientes de la excavación de su perímetro y del relevamiento efectuado de las fuentes históricas (textos, diarios, escritos de la época) y documentales (documentos de compra, planos). La información que brindaban las fuentes históricas en torno a la profundidad y a las características del fondo del citado lago (asentado directamente sobre el sedimento o sobre una marmolina como base) era muy dispar y en algunos aspectos hasta contrapuesta. Por esta razón, para poder obtener datos concretos sobre estos dos aspectos y poder resolver así algunas de estas cuestiones, se realizaron acciones específicas, que implicaron:

\footnotetext{
1 El ladrillo artesanal está manufacturado a mano, sin empleo de maquinarias que establezcan su forma de manera seriada y estandarizada. Posee una diversidad en sus dimensiones (Tabla 1) y peso, siendo en ambos casos mayores que los confeccionados a máquina (Gurcke, 1987; Schávelzon, 1991). Su composición no es homogénea, pudiéndose observar el uso de antiplásticos. El acabado o terminación de su superficie presenta a veces marcas de su proceso de manufactura, así como también la ausencia de bordes completamente lisos.
} 


\begin{tabular}{|c|c|c|c|}
\hline \multicolumn{4}{|c|}{ Ladrillos } \\
\hline \multicolumn{2}{|c|}{ Tipo de manufactura } & Manual (artesanal) & Por máquina \\
\hline \multirow{2}{*}{$\begin{array}{c}\text { Dimensiones } \\
\text { (medidas promedio) }\end{array}$} & Largo & Entre $32 \mathrm{y} 43 \mathrm{~cm}$ & $25 / 27 \mathrm{~cm}$ \\
& Ancho & Entre $14 \mathrm{y} 19,5 \mathrm{~cm}$ & $12 / 13 \mathrm{~cm}$ \\
& Espesor & Entre $3,5 \mathrm{y} 6 \mathrm{~cm}$ & $5 / 5,5 \mathrm{~cm}$ \\
\hline
\end{tabular}

Tabla 1. Comparación de las dimensiones promedio de los ladrillos artesanales y los manufacturados mediante máquina.

a. La determinación de las dimensiones totales del lago. Estas se obtuvieron mediante el registro de las mediciones de su perímetro realizado por profesionales agrónomos de la Municipalidad de Herrera y de la Universidad Tecnológica Nacional utilizando un nivel óptico y basándose en los muros o sus bases identificados arqueológicamente en cada uno de sus lados. De esta manera, se pudo conocer que el mismo originalmente poseía un largo de $180 \mathrm{~m}$ y un ancho de $120 \mathrm{~m}$. Actualmente, al estar desdibujados sus bordes y tener un menor nivel de agua, sus dimensiones son un poco menores.

b. La determinación de la profundidad del actual nivel de agua, en su parte media y en su lado oeste que coincide con el único sector que posee aún parte del muro perimetral en pie. Para ello, se navegó el lago con un bote a remo en primer lugar hasta la parte media y luego hacia su lado oeste; en dichos puntos se colocó una varilla metálica con mediciones métricas que permitieron la obtención y registro de las correspondientes profundidades. Se pudo establecer la existencia de una diferencia no muy significativa de profundidad entre la parte media $(2,16 \mathrm{~m})$ y el borde oeste del lago $(3 \mathrm{~m})$. Asimismo, la altura de aproximadamente $5 \mathrm{~m}$ que posee el sector del muro que se encuentra aún en pie, sugiere que la profundidad que posee el lago hoy en día es probablemente la mitad del nivel de agua que habría tenido en el período de su funcionamiento, cuando el nivel se encontraba próximo al borde superior del muro.

Conjuntamente con estas acciones se efectuaron tareas de investigación en el archivo y en el área de documentación de colecciones del museo, donde se pudo encontrar información referida a las compras efectuadas por la familia. Se pudo identificar un pedido realizado a Inglaterra y los comprobantes de la compra de una embarcación para ser utilizada con fines recreativos en el lago artificial. Se trataba de una embarcación de tipo Pailebot, de 50 pies de eslora (sobre cubierta), cuarenta y siete pies de largo de quilla, diez de manga y cinco y medio pies de puntal, es decir la altura desde su plan hasta la cubierta superior (Castro, 1944). Este dato posibilitó conocer el tipo de nave que fue adquirida para este espacio de ocio y poder determinar, a partir de sus medidas, la profundidad mínima que debía tener el lago para que pudiese navegar, resultando necesitar posiblemente sólo un poco más de un metro de profundidad.

c. La determinación de su conformación (materiales y forma constructiva). Esto pudo ser establecido inicialmente a través de la excavación de sondeos exploratorios en su parte interna, en sectores localizados sobre el lado este del lago, donde en la actualidad no hay agua. El trabajo se efectuó con la colaboración del personal y profesionales del museo y también de la Municipalidad de Herrera. Se realizaron cinco sondeos (Figura 5) de diferentes dimensiones, alcanzando distintas profundidades. Éstas fueron determinadas a partir del hallazgo, durante la excavación, de un estrato compuesto por la arcilla propia del lugar, momento en el cual se detenía el retiro de sedimento, asumiendo de esta manera que se había llegado al estrato natural. 


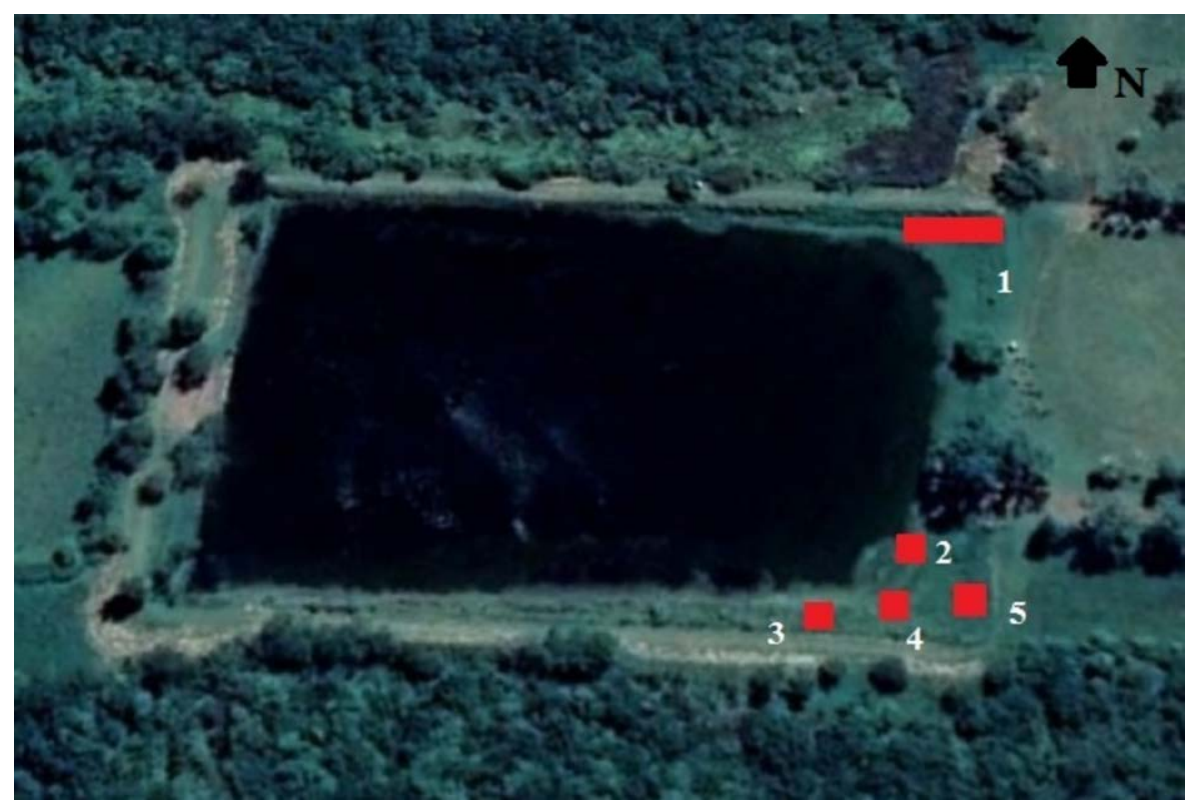

Figura 5. Localización de los sondeos en sectores internos del lago artificial que actualmente no poseen agua.

Los sondeos llevados a cabo fueron los siguientes:

1. Sondeo número 1: dimensiones de $2 \mathrm{~m}$ (orientación norte-sur) por $1,50 \mathrm{~m}$ (orientación este-oeste). Profundidad alcanzada: $2 \mathrm{~m}$.

2. Sondeo número 2: dimensiones de $2 \mathrm{~m}$ (orientación norte-sur) por $1,50 \mathrm{~m}$ (orientación este-oeste). Profundidad alcanzada: 1,60 m.

3. Sondeo número 3: dimensiones de $2 \mathrm{~m}$ (orientación norte-sur) por $3 \mathrm{~m}$ (orientación este-oeste). Profundidad alcanzada: $2,75 \mathrm{~m}$.

4. Sondeo número 4: dimensiones de 3,90 m (orientación norte-sur) por 1,60 m (orientación este-oeste). Profundidad alcanzada: 3,19 m.

5. Sondeo número 5: dimensiones de $0,85 \mathrm{~m}$ (orientación norte-sur) por 1,80 m (orientación este-oeste). Profundidad alcanzada: 1,55 m.

Para el registro de los niveles de profundidad se fijó como nivel 0 el borde del camino central de ladrillos que va desde la residencia hasta la proximidad del lago, en su lado este.

Se obtuvieron muestras de sedimento en los pozos números tres y cuatro, las que fueron llevadas al laboratorio de Construcciones civiles del Grupo de estudio de Suelos de la Universidad Tecnológica Nacional (sede Concepción del Uruguay), para su posterior análisis. Los resultados alcanzados mediante la realización de los sondeos antes mencionados evidenciaron la presencia del mismo tipo de sedimento en todos ellos con las mismas características internas. Estratigráficamente se pudo establecer que inmediatamente después de la capa de vegetación superficial se encuentra un estrato conformado por humus o tierra negra y, a continuación, otro compuesto por la denominada "tierra roja" o tierra del lugar, que se mantiene hasta el final de las profundidades alcanzadas. Esta tierra roja está conformada por una arcilla de mediana plasticidad, establecida en el Laboratorio de la Universidad Tecnológica Nacional, tomando en cuenta: el límite líquido (Norma IRAM 10501/68), el límite plástico (Norma IRAM 10502/68), el índice de plasticidad, un análisis granulométrico por vía húmeda 
(Norma IRAM 10507/69) y la clasificación de suelos por medio del Sistema Unificado de Suelos de Casagrande (Norma IRAM 10521/71).

De esta manera, mediante todas las acciones antes mencionadas, se pudo establecer que el lago tenía una pendiente que aumentaba hacia el lado oeste (la parte más alejada del Palacio), calculado esto de acuerdo a la profundidad del agua, con un muro perimetral alto en tres de sus lados (norte, sur y oeste) y bajo en su lado este, con un fondo asentado sobre una arcilla de mediana plasticidad que le proporcionaba cierto grado de impermeabilidad, impidiendo de este modo que el agua que contenía se absorbiera por completo. El nivel del agua mantenido de esta manera, se incrementaba o complementaba mediante la actividad pluvial y el ingreso de agua de una laguna cercana a través de un sistema de cañerías y bombas compradas y traídas desde el extranjero, con lo que se lograba un nivel de agua de manera permanente.

Asimismo, la investigación del perímetro y del interior del lago en forma conjunta permitió determinar que el desmoronamiento del muro perimetral se habría producido como consecuencia del desplazamiento de la arcilla que conforma el terraplén, la cual, al ser plástica y expandible, fue empujando al muro de manera constante, sin encontrar a partir de finales del siglo XIX-comienzos del siglo XX, la resistencia opuesta del agua, cuyo nivel, en los años de su funcionamiento (cuando ocupaba el palacio la familia Urquiza), era mantenido de manera permanente y era próximo al borde superior del muro. Este desplazamiento ocasionó que sólo permanecieran en pie 60 metros del muro inicial.

\section{Sector oeste del perímetro del lago}

En el lado oeste del lago (el más alejado del palacio), en un espacio intermedio entre éste y el corral, las excavaciones efectuadas permitieron hallar los cimientos de un antiguo mirador con forma de templete ${ }^{2}$, construido conjuntamente con el lago artificial entre los años 1861 y 1868. Si bien no se pudo obtener evidencia material de su parte superior, dado que ésta ha desaparecido por completo, la prolongación en altura de la forma de su base, conjuntamente con la información obtenida a partir de una fotografía tomada en el año 1875 (http://concepcionhistoriayturismo.com/2018/07/04/2189/), es decir, cinco años después del asesinato de Justo José de Urquiza y del abandono de la residencia por gran parte de sus habitantes, posibilitó observar que presentaba una forma similar a la de un templete comúnmente utilizado en los llamados parques o jardines ingleses. Este tipo de construcción ubicada por lo general en proximidad a cursos o espejos de agua, constituyó uno de los elementos del paisajismo de fines del siglo XVIII y principios del siglo XIX. Eran espacios construidos para el ocio y la recreación, insertos en un ámbito natural, rodeados por terreno circundante con vegetación, conformados por ciertos elementos comunes, tales como: estatuas, un lago $y$, alrededor de éste, un pabellón hexagonal a menudo con forma de templo (Berjman, 2018; Bonells, 2017).

Los resultados de la excavación de este sector permitieron establecer que fue construido con ladrillos de confección artesanal, con un cimiento conformado por 29 hileras de ladrillos, que alcanzan aproximadamente un metro cincuenta y cinco de profundidad. La importante base sobre la cual fue edificado posiblemente haya sido necesaria para darle estabilidad, dado que se asentaba sobre el terraplén de arcilla del lugar, la cual, como se ha mencionado anteriormente, posee cierta plasticidad que habría impedido una adecuada estabilización de la construcción sin este importante cimiento (Figura 6).

2 Es un término genérico que se aplica a construcciones pequeñas, en forma de templo que puede tener abiertos o no sus lados y que son utilizados con distintos fines. 


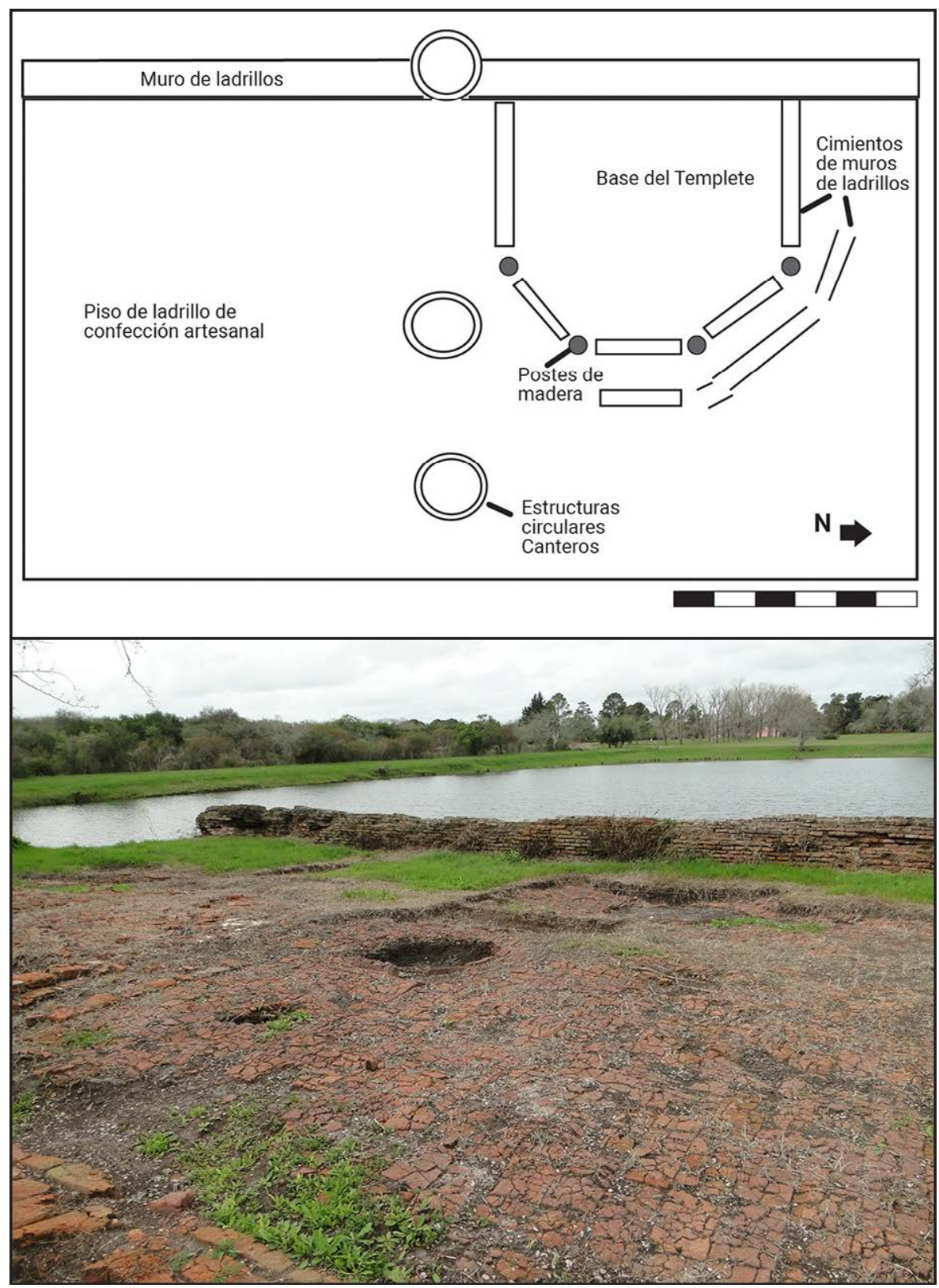

Figura 6. Croquis con la forma de la base del mirador (Templete), sus postes, el patio de ladrillos y los canteros circulares asociados (izq.). Vista del patio de ladrillos artesanales y de uno de los canteros circulares. Por detrás, el lago artificial (der.).

La forma del mirador pudo establecerse a partir de los restos hallados de su base: la misma estaba conformada por seis lados, tres de los cuales se encontraban en distintas posiciones orientadas hacia el lago, alternados con postes de madera que poseían un refuerzo metálico colocado en sus bases por debajo del piso, algo habitualmente observado en las construcciones arqueológicas del siglo XIX (por ejemplo en la construcción interna de la Aduana Nueva o Aduana Taylor de Buenos Aires). El análisis de las maderas de dichos postes fue llevado a cabo en el laboratorio de la Universidad del Museo Social Argentino, aplicando la técnica convencional de Franklin (ácido acético glacial - agua oxigenada de 100 volúmenes) y empleando la base de datos Inside Wood. Esto permitió establecer que la madera utilizada fue el Astronium balansae, llamado comúnmente Urunday. La misma es una madera autóctona de la zona, considerada de acuerdo a su ficha técnica como incorruptible, dura, pesada y 
de larga duración, siendo empleada por estas características para postes, cubiertas y durmientes (Rodríguez, 2018). El lado de la base de la estructura del templete opuesto al lago se orientaba hacia los corrales y a diferencia de su frente, estaba compuesto por una sola parte de forma recta (Figura 6).

Junto al mirador y rodeándolo, se halló un piso de ladrillos también de confección artesanal con tres estructuras circulares, cuyas dimensiones varían entre $1,20 \mathrm{~m}$ y $1,30 \mathrm{~m}$ de diámetro, las que habrían sido durante el tiempo de funcionamiento del lago como tal, grandes canteros. La forma está determinada por ladrillos puestos de canto, en forma circular y con sedimento en su interior. Estas estructuras circulares están en línea perpendicular al largo del lago y se encuentran separadas entre sí por 2,38 $\mathrm{m}$ entre la primera desde el borde del lago y la segunda y por 2,65 $\mathrm{m}$ entre ésta y la tercera.

\section{3. Área de alta densidad de restos: basural antiguo}

Hacia el lado sur del predio que rodea al Palacio, aproximadamente a $300 \mathrm{~m}$ de la residencia, la presencia de fragmentos de tipo doméstico en superficie posibilitó identificar un sector con una alta densidad de evidencia arqueológica. Este sector se encuentra formando parte de un área mucho mayor donde, a lo largo de diferentes épocas del siglo XX, se llevó a cabo el descarte de basura. Hasta el momento no se ha encontrado información histórica que haga referencia a la utilización de este lugar de descarte también en momentos anteriores.

Se efectuaron inicialmente dos sondeos exploratorios para poder determinar su potencialidad. El hallazgo de fragmentos pertenecientes al siglo XIX, motivó el planteo y ejecución de unidades de excavación en extensión y en profundidad. A partir de esta identificación, se plantearon un total de diez cuadrículas que abarcaron una superficie total de $18 \mathrm{~m}^{2}$. En los primeros $0,20 \mathrm{~m}$ de profundidad se observó una alta densidad de restos arqueológicos, decreciendo hacía los $0,30 \mathrm{~m}$ y siendo muy baja a partir de los $0,40 \mathrm{~m}$ de profundidad.

El propósito de tal excavación fue poder establecer la extensión aproximada de dicha área de descarte y el grado de potencialidad que presentaba, además de, a partir del análisis y procesamiento del material hallado y recuperado, poder determinar si pertenecía a momentos de ocupación del palacio por parte de la familia Urquiza o a épocas posteriores.

Los rasgos diagnósticos presentes en gran parte de la evidencia recuperada en los sondeos inicialmente efectuados (tipo de lozas, metales, forma, manufactura y material de las botellas, entre otros) posibilitaron ser adjudicados cronológicamente a un contexto perteneciente al siglo XIX. Se pudo determinar también que efectivamente se trataba de un sector de descarte de tipo secundario o terciario (Schiffer, 1972), entendido como un espacio en donde fue trasladada y depositada basura generada en otros espacios o contextos de uso.

En la excavación se recuperaron 1.849 restos arqueológicos (Tabla 2; Figura 7) todos ellos de características domésticas, los cuales, dada la asignación cronológica establecida a partir de la evidencia diagnóstica identificada en los sondeos iniciales, habrían sido utilizados en la vida cotidiana del lugar mientras la familia Urquiza habitaba la residencia. Entre los restos más significativos se encuentran: botellas de gres de ginebra, fragmentos de vidrios de ventanas y de botellas, flejes metálicos de barriles, lozas con decoración pintada a mano, restos óseos de animales con evidencia de consumo, una cazoleta de caolín decorada y fragmentos de tubos -todos ellos 


\begin{tabular}{|c|c|c|c|}
\hline \multicolumn{2}{|c|}{ Tipo de material } & Frecuencia total & Frecuencia \% \\
\hline \multicolumn{2}{|l|}{ Óseo } & 1.159 & 62,68 \\
\hline \multicolumn{2}{|l|}{ Metal } & 111 & 6,00 \\
\hline \multicolumn{2}{|l|}{ Vidrio } & 273 & 14,76 \\
\hline \multicolumn{2}{|l|}{ Madera } & 5 & 0,27 \\
\hline Cerámica & $\begin{array}{l}\text { Cerámica de tradición local } \\
\text { Baldosa } \\
\text { Ladrillo } \\
\text { Mayólica } \\
\text { Loza } \\
\text { Porcelana } \\
\text { Gres }\end{array}$ & $\begin{array}{c}1 \\
18 \\
26 \\
1 \\
51 \\
1 \\
201\end{array}$ & $\begin{array}{l}0,05 \\
0,97 \\
1,43 \\
0,05 \\
2,76 \\
0,05 \\
10,87\end{array}$ \\
\hline \multicolumn{2}{|l|}{ Otros } & 2 & 0,11 \\
\hline \multicolumn{2}{|l|}{ Total } & 1.849 & 100,00 \\
\hline
\end{tabular}

Tabla 2. Frecuencias totales y porcentuales de la evidencia arqueológica recuperada por tipo de material.

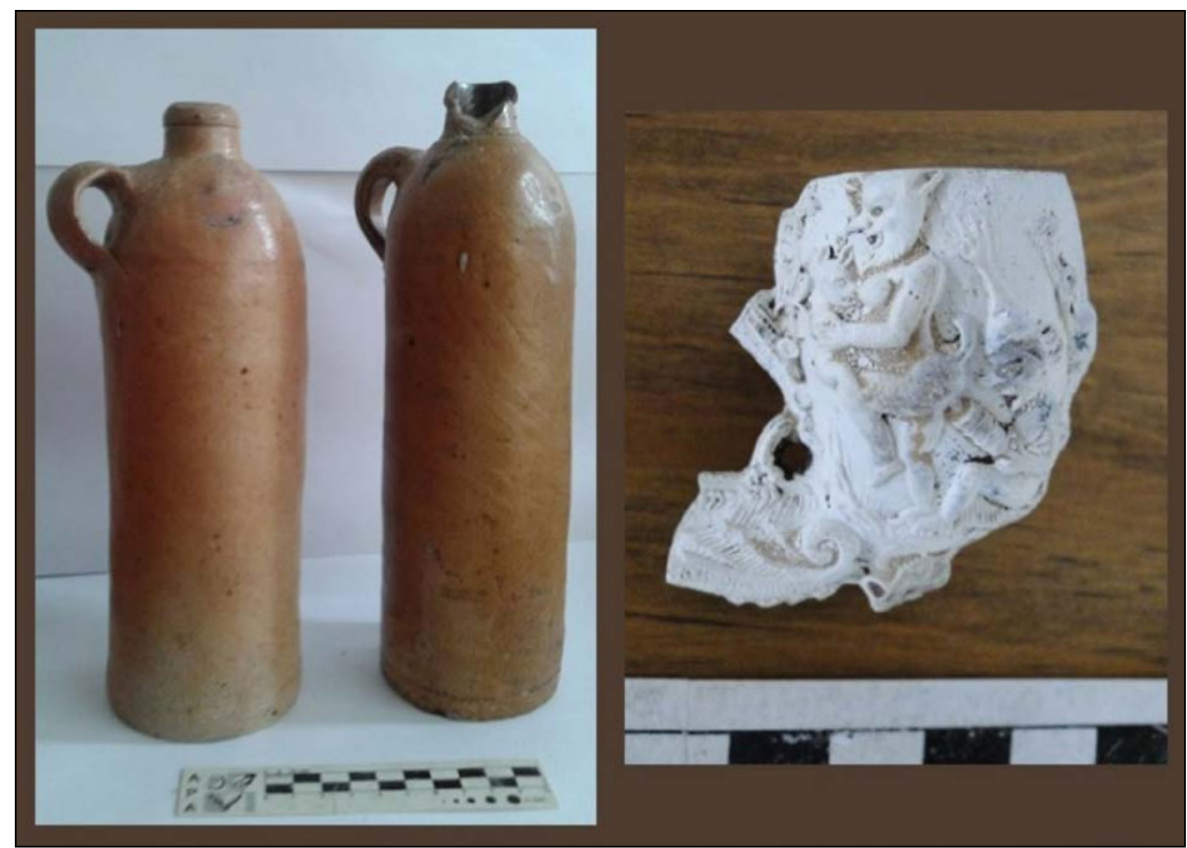

Figura 7. Objetos recuperados en la excavación del basural. Botellas de gres, de ginebra (izq.). Cazuela de una pipa de caolín para fumar tabaco con decoración tallada de diablitos (der.).

pertenecientes a pipas para fumar tabaco-, la parte final de un estuche de un sable, una parte de una cuchara y clavos metálicos de forjado artesanal, entre otros.

Estudios y análisis efectuados durante el trabajo en laboratorio y gabinete permitieron obtener información sobre ciertos aspectos de la evidencia hallada. Por ejemplo, la loza recuperada representa el $2,76 \%(n=51)$ de la muestra y en su totalidad se encuentra fragmentada. Corresponden al tipo Pearlware, de procedencia inglesa, con decoración 
pintada a mano mayormente de motivos florales. En cuanto a los restos óseos de animales, éstos poseen la mayor frecuencia porcentual de toda la muestra recuperada, constituyendo el $62,68 \%(n=1.159)$. Pudo determinarse que pertenecían a las especies Bos taurus (vaca), Ovis aries (oveja) y a Oryctolagus cuniculus (conejo), todos ellos con evidencias de haber sido consumidos, probablemente como parte de algunas de sus comidas. En el caso de los restos pertenecientes a Bos Taurus (vaca) se observa la presencia de huesos largos pertenecientes a las extremidades con presencia de fractura longitudinal intencional que lo divide en dos partes, y que se correspondería con la acción de extracción de tuétano para su consumo.

Se pudo establecer además, a partir de la evidencia recuperada, la presencia de alimentos enlatados y bebidas alcohólicas envasadas en botellas de gres (cerámica vidriada altamente resistente) y de pipas de cerámica blanca de alta pureza y dureza (de caolín) para fumar tabaco, observándose en una de ellas una decoración tallada en la misma pieza de personajes similares a diablillos y otros motivos figurativos diversos (Figura 7).

\section{Consideraciones finales}

La investigación arqueológica llevada a cabo en el Palacio San José - Museo y Monumento Histórico "Justo José de Urquiza", constituyó la primera experiencia de recuperación de su patrimonio arqueológico. Esto ha posibilitado empezar a conocer, desde la materialidad, algunos otros aspectos de la vida cotidiana de los habitantes del lugar en el siglo XIX y las características constructivas de edificaciones dedicadas al ocio y a la recreación, como fueron el lago artificial, la vereda en su perímetro y el mirador localizado en uno de sus lados. Toda la información y los datos obtenidos a partir de las excavaciones arqueológicas realizadas, complementarán los abundantes conocimientos ya existentes sobre este reconocido lugar que cuenta con una intensa y rica historia.

La posibilidad de arribar a un conocimiento inicial desde la evidencia material que permitió empezar a entender las características internas que poseía el lago en cuanto a su conformación y aspectos constructivos, constituye un primer avance sobre la temática, la que podrá ser explorada en el futuro por estudios más específicos. El conocimiento alcanzado hasta el momento permitió profundizar e ir un poco más allá de los datos brindados por las fuentes primarias y secundarias. De esta manera, se pudo comprobar que el lago tenía un declive hacia el lado oeste (el más alejado del palacio), con un muro perimetral de mampostería de ladrillos de confección artesanal y con una vereda de tipo boulevard. Ambos se encuentran apoyados sobre un terraplén artificial conformado por una arcilla característica del lugar, que también se halla en la base del lago, otorgándole cierta impermeabilidad e impidiendo así la completa absorción de su nivel de agua, el que era mantenido a su vez por el aporte pluvial y por el ingreso proveniente de una laguna cercana al sitio y próxima al río Gualeguaychú. Los resultados del análisis de esta arcilla que compone el terraplén en el laboratorio de materiales, han permitido además poder entender una de las causas de por las que los muros perimetrales no pudieron mantenerse en pie a través de los años, sobreviviendo tan sólo unos pocos metros de éste en su posición original.

Asimismo, la excavación del sector en donde se encontraba el antiguo mirador, en el lado oeste del lago, posibilitó determinar con exactitud la forma de su base y algunos de sus materiales constitutivos, aportando un nuevo dato en relación a su parte posterior, de la cual no se tenía ninguna información ni referencia histórica acerca de cómo era. La única fotografía en la que se puede observar el mirador, muestra solamente la parte de éste que se orienta hacia el lago. 
La identificación y excavación del área de descarte ha sido un hallazgo singular, de gran importancia dentro de la investigación, dado que ha permitido obtener datos acerca de la vida cotidiana de las personas que ocuparon el palacio durante el siglo XIX. Si bien gran parte de la muestra recuperada aún está en proceso de análisis para poder indagar en profundidad aspectos vinculados con el consumo interno y con la adquisición de bienes, los resultados iniciales han posibilitado observar la presencia de aquellos animales que formaron parte de la alimentación del lugar, técnicas de procesamiento de la evidencia ósea, el consumo de determinados productos (como los enlatados), así como también la presencia de objetos singulares vinculados a otras actividades no domésticas o de consumo.

La experiencia de participación con la comunidad universitaria de Concepción del Uruguay en las tareas de excavación y de laboratorio llevadas a cabo, ha sido un aspecto sustancial del proyecto de investigación arqueológica propuesto y ejecutado, propiciando poder instaurar una conciencia participativa y responsable que se vincula y actúa sobre su propio patrimonio local. Esta ha sido una primera experiencia con resultados muy satisfactorios, debido a lo cual podría aplicarse a proyectos o lugares que presentaren características similares.

Todos los estudios realizados y la información adquirida mediante la investigación arqueológica llevada a cabo han contribuido y enriquecido aún más el amplio y profundo conocimiento que ya se tiene sobre el Palacio San José. Sin duda queda mucho por realizar y mucho para contribuir también.

\section{Agradecimientos}

Al Dr. Luis Cerrudo, a la arquitecta Patricia Viganoni, al arquitecto Guillermo Minatta, a la Lic. Alejandra Heit y a todo el personal que colaboró con la investigación arqueológica, histórica y documental. A la Asociación de Amigos del Palacio San José, por el apoyo recibido. A la Dirección Nacional de Museos por permitir a la autora efectuar dicha investigación y al Ministerio de Cultura de la Nación por solventar sus pasajes y viáticos. A los rectores de la Universidad Tecnológica Nacional (sede Concepción del Uruguay), de la Universidad Autónoma de Entre Ríos y de la Universidad de Concepción del Uruguay. A Raúl Martin, jefe de Laboratorios de la Universidad Tecnológica Nacional. A la Dra. Stella Maris Rivera de la Carrera de Conservación y Restauración del Patrimonio Cultural de la Universidad del Museo Social Argentino (UMSA). Agradecimiento muy especial a todos los alumnos universitarios que voluntariamente se sumaron al trabajo arqueológico a lo largo de los años de investigación, a los profesionales del Ministerio de Cultura de la Nación y del Instituto Nacional de Antropología y Pensamiento Latinoamericano. A la Municipalidad de Herrera y al Museo de la Colonia de San Justo por sus colaboraciones. A los evaluadores anónimos que contribuyeron a mejorar el manuscrito de esta nota. 


\section{Deferencias citadas}

»Almansa Sánchez, J. (2014). Arqueología pública y gestión del patrimonio. Condenados a encontrarse. Debates de arqueología medieval, 4, 11-28.

» Benvenuto, O. (1988). El general Urquiza y la Unión Nacional (Tesis Doctoral inédita), Universidad de Buenos Aires, Argentina.

" Berjman, S. (2018). Historia delosjardines argentinos. http://www.economiayviveros. com.ar/agosto2018/plantas_ornamentales_y_flores_de_corte-cultivos_viverospaisajismo-jardineria-arte_floral-1.html. (Acceso: 5 de enero, 2020).

"Bonells, J. E. (2017). Historia de la Jardinería. https://jardinessinfronteras. com/2017/01/30/rasgos-de-la-historia-de-la-jardineria-mundial-capitulo-viii-jardiningles-de-paisaje-y-jardin-paisajista-frances/ (Acceso: 18 de diciembre, 2019).

" Castro, A. (1944). Nueva historia de Urquiza: industrial, comerciante, ganadero. Buenos Aires: Editorial Araujo.

" Cerrudo, L. A. (2000). El Palacio San José, casa del general Urquiza. Buenos Aires: Editorial Dunken.

" Gurcke, K. (1987). Bricks and Brickmaking, A Handbook for Historical Archaeology. Idaho: University of Idaho Press.

"Ley $\mathrm{N}^{\circ} 12.261 / 35$. Monumento Nacional, se declara al Palacio San José en Concepción del Uruguay, provincia de Entre Ríos. Boletín oficial de la República Argentina. $\mathrm{N}^{\circ}$ 12.394. Presidencia de la Nación.

"Lleras, R. (2018). Arqueología pública y usos sociales del patrimonio arqueológico. Trabajo presentado en las II Jornadas de Arqueología, Medellín, Colombia.

"Rodríguez, B. (2018). Urunday. http://taxonomia905b.blogspot.com/2018/11/ urunday.html. (Acceso: 20 de diciembre, 2019).

"Salerno, V. (2013). Arqueología Pública: Reflexiones sobre la construcción de un objeto de Estudio. Revista Chilena de Antropología, 27, 7-37.

"Schávelzon, D. (1991). Arqueología Histórica de Buenos Aires I. La cultura material porteña de los siglos XVIII y XIX. Buenos Aires: Corregidor.

"Schiffer, M. B. (1972). Archaelogical context and systemic context. American Antiquity, 37(2), 156-165.

"Tapella, E. (2007). El mapeo de Actores claves, documento de trabajo del proyecto Efectos de la biodiversidad funcional sobre procesos ecosistémicos, servicios ecosistémicos y sustentabilidad en las Américas: un abordaje interdisciplinario. https://planificacionsocialunsj.files.wordpress.com/2011/09/quc3a9-es-elmapeo-de-actores-tapella1.pdf (Acceso: 19 de mayo, 2020). 